\title{
Effects of Catalyst Concentration and Residence Time on Transesterification of Palm Oil with Methanol Using a 1.0 mm ID Millichannel Reactor
}

\author{
Mohd Azam Nurul AsYikin*1, Yoshimitsu UemurA*1†, \\ Katsuki KuSAKABE *2, and Bustam MOHAMAD AZMI*3
}

(Received February 17, 2016)

\begin{abstract}
Millichannel reactor designed for continuous reaction of transesterification has been studied in recent years and also attracted the focus of scientists for its capability to produce high quality biodiesel yield. The millichannel reactor exhibits many advantages, one of which includes great improvement in reaction rate, product yield, energy efficiency as well as mass and heat transfer. Therefore, the millichannel reactor has a high potential to be used for biodiesel production. In this study, transesterification of refined palm oil was carried out by using a teflon tube with an internal diameter of $1.0 \mathrm{~mm}$ at 21:1 methanol to oil molar ratio and $60^{\circ} \mathrm{C}$ to investigate the effects of potassium hydroxide catalyst concentration $(0.5,1.0,1.5,2.0,2.5,3.0,3.5,4.0,4.5$, and $5.0 \mathrm{wt} \%)$, and residence time $(60,120$, and 180 s) on the oil conversion and fatty acid methyl ester (FAME) yield. The transesterification using a batch reactor was also performed to compare with the millichannel reactor at conditions: 21:1 methanol to oil molar ratio, temperature of $60^{\circ} \mathrm{C}$ and $0.5 \mathrm{wt} \%$ potassium hydroxide concentration. Besides that, the process time has been varied in the ranges of 60,120 , and $180 \mathrm{~s}$. In this study, the best FAME yield was obtained at $4.5 \mathrm{wt} \%$ catalyst concentration and residence time of $180 \mathrm{~s}$. As a result, the millichannel reactor revealed a higher oil conversion and FAME yield compared to the batch reactor.
\end{abstract}

Key Words

FAME yield, Millichannel reactor, Oil conversion, Transesterification

\section{Introduction}

Biodiesel, a biodegradable and non-toxic fuel, was produced from renewable sources such as vegetable oils and animal fats. Generally, biodiesel can be produced via a supercritical methanol process, transesterification process, pyrolysis process, as well as blending and microemulsion process. However, the common process used to convert triglycerides to biodiesel is the transesterification process ${ }^{1)}$, which is a process whereby the triglyceride reacts with

\footnotetext{
※ Centre for Biofuel and Biochemical Research (CBBR), Universiti Teknologi PETRONAS 32610 Bandar Seri Iskandar, Perak, Malaysia

※2 Department of Nanoscience, Sojo University Kumamoto 860-0082, Japan

※3 Department of Chemical Engineering, Universiti Teknologi PETRONAS 32610 Bandar Seri Iskandar, Perak, Malaysia

$\dagger \quad$ Corresponding author: yoshimitsu_uemura@utp.edu.my
}

methanol in the presence of a catalyst, which could be either acidic or basic depending on the type of feedstock used ${ }^{2)}$. Nonetheless, Ma et al. ${ }^{2)}$ reported that the alkali catalysts were found to be more effective. Nevertheless, the choice of acid and alkali catalysts used depends on the free fatty acids (FFA) content in the feedstock.

Transesterification process between vegetable oil and methanol is an immiscible reaction. Therefore, the millichannel reactor is recommended for biodiesel production, as it can improve the reaction between immiscible liquids ${ }^{3}$. Furthermore, the required reaction time can be reduced and a better enhancement in mass transfer is also achieved by using the millichannel reactor compared to a batch reactor ${ }^{4)}$. Guan et al. ${ }^{4)}$ stated that, by using a millichannel reactor, 100\% oil conversion was achieved at 240 s, while $100 \%$ of oil conversion was only achieved at $600 \mathrm{~s}$ when using a batch reactor with similar 
parameters. Moreover, Rashid et al. ${ }^{5)}$ also conducted an experiment using the millichannel reactor and acquired results showing that the millichannel reactor produced high FAME yield at a longer tube length and slower flow rate.

In addition, the small dimensions of the millichannel reactor provide a lot of benefits to the biodiesel production, which include minimising the amount of reactants, increasing the surface area to volume ratio, and low energy consumption. Therefore, with the aim of using it in industrial application, different sizes of tube reactors need to be investigated.

For this research, an experimental study was conducted to investigate the effects of potassium hydroxide catalyst concentration (0.5-5.0 wt\%) and residence time (60180 s) on the oil conversion and FAME yield. Meanwhile, the transesterification process using the batch reactor was also conducted for the comparison with the millichannel reactor at conditions of: 21:1 methanol to oil molar ratio, temperature at $60^{\circ} \mathrm{C}$, and $0.5 \mathrm{wt} \%$ potassium hydroxide $(\mathrm{KOH})$ concentration.

\section{Experiment}

The refined palm oil (feedstock) was purchased from FFM Berhad, Malaysia, while the methanol, potassium hydroxide, and acetic acid were purchased from Merck Co. Ltd. Transparent polytetrafluoroethylene (PTFE) tubes (with inner diameter $=1.0 \mathrm{~mm}$ and length=1000 mm) were used for the millichannel reactor.

Fig. 1 shows the schematic diagram of the millichannel reactor system ${ }^{6}$. Two syringe pumps were used to inject the oil and methanol containing $\mathrm{KOH}$ respectively. The residence time for reaction varied from 60 to $180 \mathrm{~s}$. The flow rate of oil was $8.2 \mathrm{~mL} / \mathrm{h}$ and the flow rate of methanol was $7.5 \mathrm{~mL} / \mathrm{h}$ for the case of $180 \mathrm{~s}$ of residence time. Maintaining this ratio, total flow rate was $47.12 \mathrm{~mL} / \mathrm{h}$ for $60 \mathrm{~s}$ of residence time, $23.58 \mathrm{~mL} / \mathrm{h}$ for $120 \mathrm{~s}$ of residence time, and $15.70 \mathrm{~mL} / \mathrm{h}$ for $180 \mathrm{~s}$ of residence time. Both oil and methanol containing $\mathrm{KOH}$ were mixed at a T-shape joint before flowing into the millichannel reactor, which was placed on a hot plate and covered by a glass plate to maintain the temperature at $60^{\circ} \mathrm{C}$, as well as to prevent the heat from releasing into the surroundings, thus trapping the heat inside the reactor. The $\mathrm{KOH}$ concentration was changed in the range of $0.5-5 \mathrm{wt} \%$. Theoretically, one mol of acetic acid is required to neutralise one mol of $\mathrm{KOH}$ catalyst. Therefore, $10 \%(\mathrm{v} / \mathrm{v})$ of the acetic acid $(40 \mathrm{~mL})$ was used to terminate the reaction of the product. The bottle containing the products and acetic acid was shaken by hand to assure complete mixing. The upper FAME layer was separated and rinsed with deionised water three times using centrifuge at $4500 \mathrm{rpm}$ for $15 \mathrm{~min}$ to remove all the residues.

The experiment was also carried out by using a batch reactor (Fig. 2). A $250 \mathrm{~mL}$ glass, round-bottomed flask with three necks with dimensions 14 and $24 \mathrm{~mm}$ of inner diameter and 23 and $29 \mathrm{~mm}$ of outer diameter was used. The thickness of the glass, round-bottomed flask beaker as shown in Fig. 2 is $2.67 \mathrm{~mm}$. With the glass, round-bottomed flask connected to the condenser, a thermocouple was used to detect the temperature, while a magnetic stirrer was used to mix the reactants. Refined palm oil (40 g) was placed in the reactor and heated in a water bath at $60^{\circ} \mathrm{C}$. When the reactor reached the set temperature, $32.02 \mathrm{~g}$ of methanol containing $0.5 \mathrm{wt} \%$ of $\mathrm{KOH}$ concentration was added into the reactor and stirred at $300 \mathrm{rpm}$. The molar

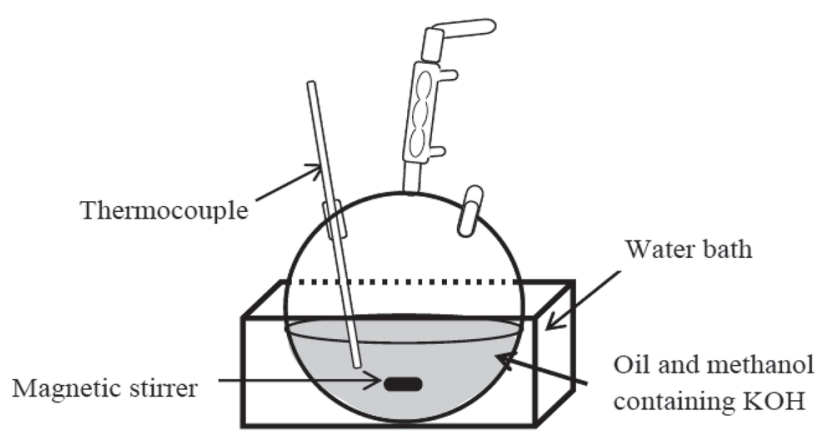

Fig. 2 Batch reactor system

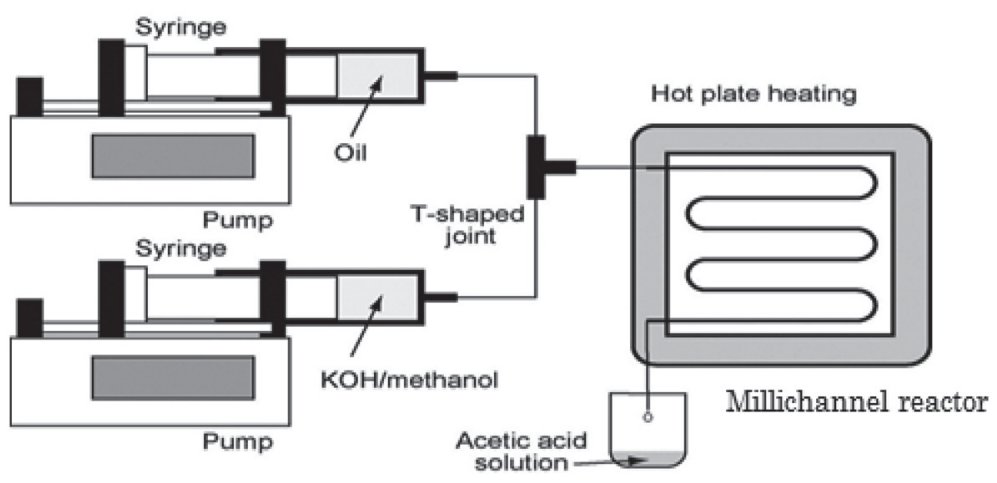

Fig. 1 Millichannel reactor system 
ratio for methanol to oil was fixed at 21:1. The reaction time were 60, 120 and $180 \mathrm{~s}$. The product collected (72 g) was then placed in a beaker containing $40 \mathrm{~mL}$ of acetic acid aqueous solution with concentration $10 \%(\mathrm{v} / \mathrm{v})$. The upper FAME layer was separated and rinsed three times with deionised water and centrifuged at $4500 \mathrm{rpm}$ for $15 \mathrm{~min}$ with the purpose of removing impurities.

The products were analysed using a High Performance Liquid Chromatography (HPLC) from the Shimadzu Prominence equipped with a silica-gel column (Shimpack CLC-SIL) and a refractive index detector. The mobile phase used was n-hexane/2-propanol with 99.5/0.5 (v/v) ratio. The column temperature was set at $40{ }^{\circ} \mathrm{C}$ with a flow rate of $1 \mathrm{~mL} / \mathrm{min}$. For the sample preparation, $100 \mu \mathrm{L}$ of the product was diluted in $3 \mathrm{~mL}$ of $\mathrm{n}$-hexane ${ }^{5)}$. Based on the analysis by HPLC, two peaks appeared in the chromatogram, representing the sum of the unreacted glyceride (monoglyceride, diglyceride, and triglyceride) and sum of the FAME yielded, respectively ${ }^{7}$. The FAME yield YFAME was calculated as ${ }^{8)}$ :

$$
Y_{F A M E}=\frac{C_{F A M E}}{3 C_{0}}
$$

where $C_{F A M E}\left[\mathrm{~mol} / \mathrm{m}^{3}\right]$ is the FAME concentration in the product and $C_{0}\left[\mathrm{~mol} / \mathrm{m}^{3}\right]$ is the concentration of the triglycerides in the feedstock. For volume to calculate the concentration, sum of the volume of oil and methanol was used. In this study, $C_{0}$ was $560 \mathrm{~mol} / \mathrm{m}^{3}$.

Meanwhile, the oil conversion $X_{\text {oil }}$ that represented the fraction of the triglycerides that was converted into the FAME and some unknown compounds were calculated as ${ }^{7)}$ :

$$
X_{\text {oil }}=\frac{C_{0}-C}{C_{0}}
$$

where $C_{0}\left[\mathrm{~mol} / \mathrm{m}^{3}\right]$ and $C\left[\mathrm{~mol} / \mathrm{m}^{3}\right]$ represent the concentration of the triglycerides before and after the reaction in the reaction system.

Reaction rate $r\left[\mathrm{~mol} /\left(\mathrm{m}^{3} \mathrm{~s}\right)\right]$ was calculated using difference of oil concentration for the different residence time as:

$$
r=-\frac{\Delta C}{\Delta \tau}
$$

where $\Delta C[\mathrm{~mol} / \mathrm{m} 3]$ and $\Delta \tau[\mathrm{s}]$ represent the difference of oil concentration in the product and the corresponding difference of residence time, respectively.

\section{Results and Discussion}

To reduce the residence time and obtain high oil conversion and FAME yield, the catalyst concentration must be optimised. Fig. 3 shows the effect of catalyst concentration on the oil conversion and FAME yield. In this study, as the free fatty acid content is low around

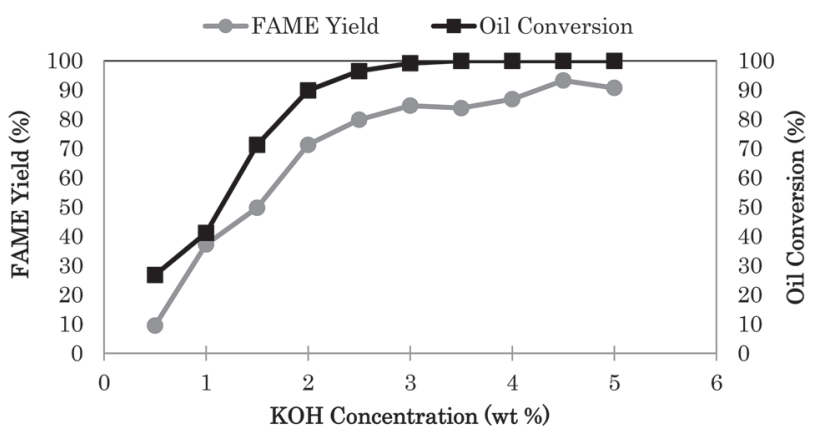

Fig. 3 Effect of the catalyst concentration on the oil conversion and FAME yield (methanol to oil molar ratio: 21:1, temperature: $60^{\circ} \mathrm{C}$, residence time: $180 \mathrm{~s}$, total flow rate of $15.70 \mathrm{ml} / \mathrm{h}$ )

0.065\%, only one step transesterification process was done in agreement with the statement by Tanawannapong et al. ${ }^{9}$. One step transesterification process is the process whereby only alkali catalyst was used.

The oil conversion and FAME yield increase when the potassium hydroxide catalyst concentration was also increased. The result from Fig. 3 shows that the best FAME yield was obtained at $4.5 \mathrm{wt} \%$ catalyst concentration and lowest at $0.5 \mathrm{wt} \%$. However, at $5 \mathrm{wt} \%$ catalyst concentration, the FAME yield decreased slightly, indicating that the $4.5 \mathrm{wt} \%$ is an optimum concentration of $\mathrm{KOH}$ for this system, while the $5 \mathrm{wt} \% \mathrm{KOH}$ and above might have caused soap formation ${ }^{9)}{ }^{10}$. This finding is in a fair agreement with the results reported by Sun et al. ${ }^{11}$.

Fig. 4a illustrates the effect of residence time on the oil conversion and FAME yield. In this experiment, 4.5 wt\% concentration of $\mathrm{KOH}$ was used as an optimum catalyst concentration. The residence times were varied from 60 to $180 \mathrm{~s}$ and the results in Fig. 4a showed that the FAME yield increased from $67 \%$ to $93 \%$ with an increase in residence time from 60 to $180 \mathrm{~s}$. The oil conversion increased from 60 to $120 \mathrm{~s}$ and increased slightly at $180 \mathrm{~s}$. Thus, the best FAME yield was observed at $180 \mathrm{~s}$ because, at a longer reaction time, both reactants had sufficient time to diffuse and complete the reaction. The findings showed that the FAME yield increased with the residence time, which is similar to the results obtained by Sun et al. ${ }^{11}$.

Fig. $4 \mathrm{~b}$ depicts the plot of reaction rate. It could be clearly seen that $180 \mathrm{~s}$ of residence time had the lowest reaction rate that represented $100 \%$ oil conversion. This was mainly due to the fully consumption of the reactant (oil). Since all the reactant undergo completed reaction, the reaction rate is lowest at $180 \mathrm{~s}$. Therefore, $180 \mathrm{~s}$ is the optimum residence time for this study. Nonetheless, there are a few types of flow patterns, such as the annular flow and slug flow ${ }^{5)}$. The slug flow was observed in this research 


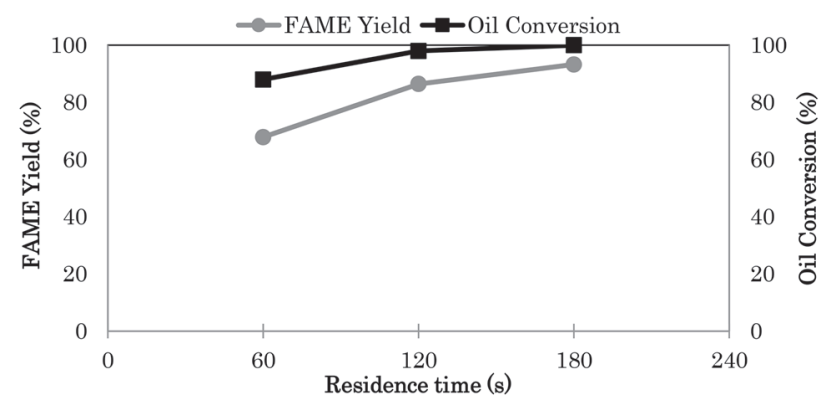

Fig. 4a Effect of residence time on the oil conversion and FAME yield (methanol to oil molar ratio: $21: 1$, temperature: $60{ }^{\circ} \mathrm{C}$, $\mathrm{KOH}$ concentration: $4.5 \mathrm{wt} \%)$

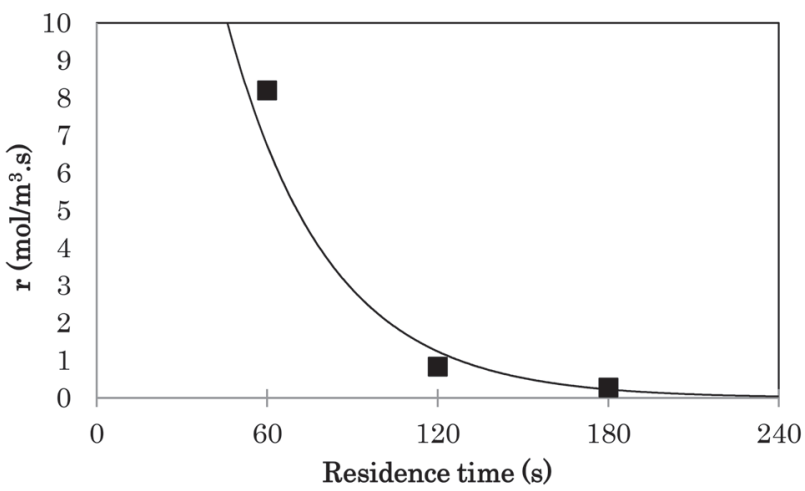

Fig. 4b Plot of reaction rate

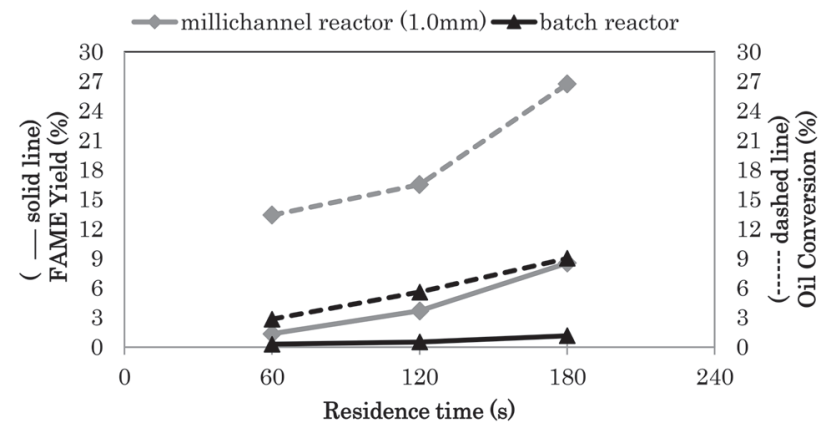

Fig. 5 Effect of residence time on the oil conversion and FAME yield for two different reactors (methanol to oil molar ratio: 21:1, temperature: $60^{\circ} \mathrm{C}, \mathrm{KOH}$ concentration: $0.5 \mathrm{wt} \%$ )

for all residence times, where the aqueous phase that represented methanol existed as a slug and was separated by organic phase (oil phase).

Fig. 5 displays the effect of residence time on the oil conversion and FAME yield of two different reactors. The 0.5 wt\% catalyst concentration that produced the lowest FAME yield was chosen to be studied to identify the effect of this parameter, based on the findings obtained from Fig. 3. If the optimised condition was used for both reactors, it could be difficult to see the trends of the results, as the oil conversion could be around 100\%. Fig. 5 shows that both reactors demonstrated the increment of FAME yield and oil conversion when the residence time increased. Nevertheless, the millichannel reactor gave the best yield compared to the batch reactor. The result may be attributed to internal circulation flow in the methanol droplets for the millichannel reactor, which increased mass transfer of the reactant triglyceride in methanol droplets.

\section{Conclusion}

Biodiesel production has been carried out using a millichannel reactor made of PTFE with a $1.0 \mathrm{~mm}$ internal diameter. It was found that oil conversion and FAME yield was influenced by catalyst concentration and residence time. Increasing the catalyst concentration could increase the FAME yield, but a very high catalyst concentration could decrease it. In this study, 4.5 wt\% concentration catalyst and $180 \mathrm{~s}$ residence time were found to be the optimum parameter, which produced the FAME yield closest to $100 \%$. Comparatively, it was found that the required residence time in the millichannel reactor was less than what was needed by the batch system to obtain the same oil conversion and FAME yield under the same reaction conditions.

\section{Acknowledgements}

The work was financially supported by the Mitsubishi Corporation Education Trust Fund and the YUTP Grant of UTP. The authors wish thanks to Dr. Fathelrahman Hamid Elhassan of the Centre for Biofuel and Biochemical Research (CBBR) for the assistance of the manuscript preparation.

\section{References}

1) Gerpen, J. V., Fuel Process. Technol., 86, 1097-1107 (2005)

2) Maa, F.; Hanna, M. A., Bioresour. Technol., 70, $1-15$ (1999)

3) Guan, G.; Teshima, M.; Sato, C.; Son, S. M.; Irfan, M. F.; Kusakabe, K., AIChE Journal, 56, 1383-1390 (2010)

4) Guan, G.; Kusakabe, K.; Moriyama, K.; Sakurai, N., Chem. Eng. Trans., 14, 237-244 (2008)

5) Rashid W. N. W. A.; Uemura, Y.; Kusakabe, K.; Osman, N. B.; Abdullah, B., J. Jpn. Inst. Energ, 92, 905-908 (2013)

6) Azam, N. A. M.; Uemura, Y.; Kusakabe, K.; Bustam, M. A., Aust. J. Basic \& Appl. Sci., 9, 177-180 (2015)

7) Guan, G.; Kusakabe, K.; Moriyama, K.; Sakurai, N., Chem. Eng. Journal, 146, 302-306 (2009)

8) Rashid W. N. W. A.; Uemura, Y.; Kusakabe, K.; Osman, N. B.; Abdullah, B., Procedia Chemistry, 9, 165-171 (2014)

9) Tanawannapong, Y.; Kaewchada, A.; Jaree, J., Ind. Eng. Chem., 19, 37-41 (2013)

10) Atadashi, I. M.; Aroua, M. K.; Aziz, A. R. A.; Sulaiman, N. M. N., Renewable Sustainable Energy Rev., 16, 3456-3470 (2012)

11) Sun, J.; Ju, J.; Ji, L.; Zhang, L.; Xu, Z., Ind. Eng. Chem. Res, 47, 1398-1403 (2007) 